\title{
A New Design Method of High-Order Modified Repetitive Control Systems for Reference Inputs with Uncertain Period-Time
}

\author{
Zhongxiang Chen, Kou Yamada, and Tatsuya Sakanushi \\ Department of Mechanical System Engineering, Gunma University, Kiryu 376-8515, Japan \\ Correspondence should be addressed to Zhongxiang Chen; t10802277@gunma-u.ac.jp
}

Received 20 October 2012; Revised 5 January 2013; Accepted 9 January 2013

Academic Editor: Zhan Shu

Copyright (C) 2013 Zhongxiang Chen et al. This is an open access article distributed under the Creative Commons Attribution License, which permits unrestricted use, distribution, and reproduction in any medium, provided the original work is properly cited.

\begin{abstract}
This paper considers the design of high-order modified repetitive control systems for periodic reference inputs with uncertain period-time. The objective of this work was to develop a new design method so that the closed-loop high-order modified repetitive control system is robustly stable with high control precision for periodic reference inputs with uncertain periodtime. The parametrization of all stabilizing controllers containing three free parameters is proposed based on the Youla-Kucera parameterization. Moreover, to obtain the free parameters, the constraint conditions were converted into stability conditions in the form of Bilinear Matrix Inequalities that can be solved using the available software. In addition, the high control precision is guaranteed by designing the free parameters after the control characteristic of this control system. The validity and effectiveness of the proposed design method were verified by numerical examples.
\end{abstract}

\section{Introduction}

In practical applications, many control systems, such as industrial robots, computer disk drives, and rotating machine tools, have to deal with periodic reference or disturbance signals. For this class of systems, Hara et al. [1] proposed a repetitive controller that applies the internal model principle [2], which states that if a reference or disturbance signal can be regarded as the output of an autonomous system, including this system in a stable feedback loop guarantees asymptotically tracking or rejecting performance. This control technique has been proven to be an efficient control scheme for handling periodic signals and has been applied extensively.

Generally, most repetitive controller designs in the literature suffer from two major drawbacks. One is the requirement of exact knowledge of the period-time of reference or disturbance signals [3]. This means that in practical applications, either the period-time is required to be a constant or an accurate measurement of the periodicity is necessary. The other drawback is due to the Bode sensitivity integral [4]: the perfect reduction at harmonic frequencies is counteracted by amplification of noise at intermediate frequencies.

To address these problems, the so-called high-order repetitive control has been established [5-13]. Inoue [6] first proposed the high-order repetitive controller to improve the control precision and cancel out the phase lag. Based on that work [6], Chang et al. [7] provided an optimization method to determine the weighting factors by minimizing the infinity norm of the relative error transfer function for the high-order repetitive controller. For small variations in the period-time, in [8], an approach is presented to design a high-order repetitive controller such that robustness for period changes is obtained. Inspired by the results in [7], Steinbuch et al. $[9,10]$ proposed a unified framework able to reproduce the results of both [7] and [8]. In contrast with the conventional design approaches that are focused on the perfect nominal periodic performance of the closed-loop system, Pipeleers et al. [5, 11] presented a systematic, semidefinite programming-based scheme to compute high-order repetitive controllers, which yields an optimal trade-off between nonperiodic performance and robust periodic performance. 
For multiple-period systems that have a similar structure to the high-order repetitive control system, solutions are given by Yamada et al. [12] and Yamada et al. [13] using the YoulaKucera parametrization (YKP) without solving the problem of designing the free parameters.

In this paper, we consider the problem of designing a high-order modified repetitive control system to track or reject external signals with uncertain period-time.

A new structure of high-order modified repetitive controller was established inspired by the conventional highorder repetitive controller. Two useful methodologies, the YKP and the Bilinear Matrix Inequalities (BMIs) approach, were adopted to design compensators in the high-order modified repetitive control system. The objective was to develop a new and simple design method that not only provides good control performance while preserving the system's stability, but can also be efficiently implemented with available software. The approach taken in this paper was as follows. First, the parametrization of all stabilizing controllers was derived on the basis of the YKP. Next, the constraint conditions of the free parameters were transformed into stability conditions in the form of BMIs. In addition, according to the control characteristic, a low-pass filter was introduced to specify the intermediate frequencies. Finally, numerical examples for a single-input/single-output (SISO) tracking system were specified to illustrate the validity and effectiveness of the proposed design method. The results presented in this paper show that the high-order modified repetitive control system can provide high control precision for periodic signals with a certain vibration in period-time. Moreover, the control precision at the intermediate frequencies was satisfied by modifying the cutoff frequency of low-pass filters in compensators.

Notations. $R H_{\infty}$ denotes the set of stable proper real rational functions; $H_{\infty}$ denotes the set of stable causal functions; $R^{n}$ denotes the $n$-dimensional Euclidean space; $R^{n \times m}$ is the set of all $n \times m$ real matrices; $I$ is the identity matrix; the null matrix or null vector of appropriate dimension is denoted by 0 ; the superscript " $T$ " stands for the transpose of a matrix; $\bar{\sigma}\{\cdot\}$ denotes the largest singular value of $\{\cdot\}$; the symmetric terms in a symmetric matrix are denoted by $*$; for example, $\left[\begin{array}{ll}A & B \\ * & C\end{array}\right]=\left[\begin{array}{cc}A & B \\ B^{T} & C\end{array}\right]$

\section{Problem Formulation}

Figure 1 depicts the proposed high-order modified repetitive control system, where $G(s) \in R^{p \times m}(s)$ is a strictly proper plant with an assumption that $p \leq m . C_{1}(s) \in R^{m \times p}(s)$ is a forward compensator to enhance the robust stability of this closed-loop system and improve the transient response, $C_{2}(s) \in R^{m \times p}(s)$ is typically called the learning filter and is used to compensate the high-order modified repetitive controller $C_{r}(s), y(t) \in R^{p}$ is the control output, and $r(t) \epsilon$ $R^{p}$ is a periodic reference input. Uncertainty of period-time is modeled as relative uncertainty on $L$, bounded by $\Delta$ :

$$
L_{\delta}:=L(1+\delta), \quad|\delta| \leq \Delta<\infty
$$

In Figure 1, the structure of the high-order modified repetitive controller $C_{r}(s)$ consists of multiple periodic signal generators and can be expressed as

$$
\begin{gathered}
C_{r}(s)=H(s)(I-H(s))^{-1}=(I-H(s))^{-1} H(s), \\
H(s)=\sum_{i=1}^{N} W_{i} q^{i}(s) e^{-s L_{i}}, \\
L_{i}=i \times L,
\end{gathered}
$$

where $W_{i}(i=1, \ldots, N)$ are the weighting factors to modify the dynamic response in between the harmonic frequencies, and $q(s) \in R^{p \times p}(s)$ is a low-pass filter assumed as a diagonal matrix function.

Like the current high-order repetitive control design approaches in $[5,7,10]$, this work relied on the following two nonrestrictive assumptions.

Assumption 1. All harmonics $l=0, \ldots, \hbar$ of the reference input are assumed to lie in the passband of $q(s)$. This can be guaranteed by the proper design of $q(s)$, provided that all harmonics $l=0, \ldots, \hbar$ lie well below $\omega_{c o}$, the cutoff frequency of $q(s)$.

Assumption 2. In its passband, $q(s)$ is assumed to equal its DC gain $q(j \omega)=I$ for all $\omega<\omega_{c o}$. These two assumptions imply that

$$
\begin{aligned}
C_{r}(s)=\widehat{C}_{r}(s) & :=(I-\widehat{H}(s))^{-1} \widehat{H}(s), \\
\widehat{H}(s) & =\sum_{i=1}^{N} W_{i} e^{-s L_{i}},
\end{aligned}
$$

for all $s=j \omega_{l}(l=0, \ldots, \hbar)$, where $L_{i}(i=1, \ldots, N)$ are defined in $(4)$ and $\omega_{l}(l=0, \ldots, \hbar)$ are the frequency components of the periodic reference input described as

$$
\omega_{l}:=\frac{2 l \pi}{L}, \quad l=0, \ldots, \hbar
$$

In this case, the $N$ weighting factors $W_{i}(i=1, \ldots, N)$ can be determined by using the methods proposed in $[5,7,10]$.

In addition, suppose that the strictly proper plant, $G(s)$, is controllable and observable and its state-space description is

$$
\begin{gathered}
\dot{x}(t)=A_{p} x(t)+B_{p} u(t), \\
y(t)=C_{p} x(t),
\end{gathered}
$$

where $A_{p} \in R^{n \times n}, B_{p} \in R^{n \times m}$, and $C_{p} \in R^{p \times n}$ are system matrices, and $x(t) \in R^{n}, u(t) \in R^{m}$, and $y(t) \in R^{p}$ denote state, control input, and control output, respectively.

The design problem considered in this paper can be stated as follows: for a given strictly proper plant $G(s)$, a constant $N$, and a low-pass filter $q(s)$, find the admissible compensators $C_{1}(s)$ and $C_{2}(s)$ such that the controller $C(s)$ written as

$$
C(s)=C_{1}(s)+C_{2}(s) C_{r}(s)
$$

robustly stabilizes the closed-loop system with high control precision for the periodic reference input with uncertainty in period-time, where $C_{r}(s)$ is defined in (2). 


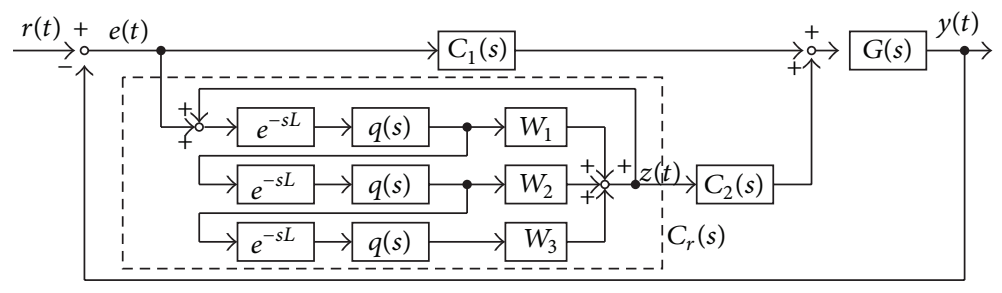

FIGURE 1: High-order modified repetitive control system for $N=3$.

\section{High-Order Modified Repetitive Control System Design}

3.1. The Parametrization of All Stabilizing Controllers. In this section, we derive the parametrization of all stabilizing controllers, with the structure of the high-order modified repetitive controller for periodic reference inputs with uncertain period-time. The necessary and sufficient conditions for the parametrization of all stabilizing controllers by means of the YKP are proposed by the following main theorem.

Theorem 1. The control system in Figure 1 is internally stable if and only if the controllers can be written as

$$
\begin{gathered}
C(s)=\left\{(Y(s)-Q(s) \widetilde{N}(s))^{-1}(X(s)+Q(s) \widetilde{D}(s)):\right. \\
|Y(\infty)-Q(\infty) \widetilde{N}(\infty)| \neq 0\} \\
=\left\{(\widetilde{X}(s)+D(s) Q(s))(\widetilde{Y}(s)-N(s) Q(s))^{-1}:\right. \\
Q \widetilde{Y}(\infty)-N(\infty) Q(\infty) \mid \neq 0\} \\
Q(s)=\left\{Q_{0 n}(s)+\left(Y(s) \widetilde{Q}(s)-Q_{0 n}(s)\right) H(s)\right\} \\
\times\left\{Q_{0 d}(s)+\left(\widetilde{N}(s) \widetilde{Q}(s)-Q_{0 d}(s)\right) H(s)\right\}^{-1} \in H_{\infty},
\end{gathered}
$$

where $Q_{0 n}(s), Q_{0 d}(s), \widetilde{Q}(s) \in R H_{\infty}$, and rank $Q_{0 d}(s)=p$, $N(s), D(s), \widetilde{N}(s)$, and $\widetilde{D}(s)$ are coprime factors of $G(s)$ on $R H_{\infty}$, and $X(s), Y(s), \widetilde{X}(s), \widetilde{Y}(s) \in R H_{\infty}$ satisfy

$$
\begin{aligned}
& {\left[\begin{array}{cc}
Y(s) & X(s) \\
-\widetilde{N}(s) & \widetilde{D}(s)
\end{array}\right]\left[\begin{array}{cc}
D(s) & -\widetilde{X}(s) \\
N(s) & \widetilde{Y}(s)
\end{array}\right]} \\
& \quad=\left[\begin{array}{cc}
D(s) & -\widetilde{X}(s) \\
N(s) & \widetilde{Y}(s)
\end{array}\right]\left[\begin{array}{cc}
Y(s) & X(s) \\
-\widetilde{N}(s) & \widetilde{D}(s)
\end{array}\right]=\left[\begin{array}{ll}
I & 0 \\
0 & I
\end{array}\right] .
\end{aligned}
$$

Proof (Necessity). To prove the necessity, suppose that there exist admissible compensators $C_{1}(s)$ and $C_{2}(s)$, such that the controller $C(s)$ with a high-order repetitive control structure written as (9) stabilizes the closed-loop system. By means of the YKP [14], the parametrization of all stabilizing controllers
$C(s)$ can be written as (10a) and (10b). Substitute $C(s)$ in (9) into (10a) and the $Q(s)$ in (11) can be represented as

$$
\begin{aligned}
Q(s)=\{ & \left(Y(s) C_{1}(s)-X(s)\right) \\
& \left.+\left(Y(s) C_{2}(s)-Y(s) C_{1}(s)+X(s)\right) H(s)\right\} \\
\times & \left\{\left(\widetilde{D}(s)+\widetilde{N}(s) C_{1}(s)\right)\right. \\
& \left.+\left(\widetilde{N}(s) C_{2}(s)-\widetilde{N}(s) C_{1}(s)-\widetilde{D}(s)\right) H(s)\right\}^{-1} .
\end{aligned}
$$

Let $C_{1 n}(s) \in R H_{\infty}$ and $C_{1 d}(s) \in R H_{\infty}$ be coprime factors of $C_{1}(s)$ on $R H_{\infty}$ satisfying

$$
C_{1}(s)=C_{1 n}(s) C_{1 d}^{-1}(s) .
$$

Under the assumptions in Section 2, the low-pass filter $q(s)$ is a diagonal matrix function; this means that $H(s)$ is also a diagonal matrix function, and substituting (14) into (13) yields

$$
\begin{aligned}
Q(s)=\{ & \left(Y(s) C_{1 n}(s)-X(s) C_{1 d}(s)\right) \\
& +\left(Y(s) C_{2}(s) C_{1 d}(s)-Y(s) C_{1 n}(s)\right. \\
& \left.\left.+X(s) C_{1 d}(s)\right) H(s)\right\} \\
\times & \left\{\left(\widetilde{D}(s) C_{1 d}(s)+\widetilde{N}(s) C_{1 n}(s)\right)\right. \\
& +\left(\widetilde{N}(s) C_{2}(s) C_{1 d}(s)\right. \\
& \left.\left.\quad-\widetilde{N}(s) C_{1 n}(s)-\widetilde{D}(s) C_{1 d}(s)\right) H(s)\right\}^{-1} .
\end{aligned}
$$

Moreover, let $C_{2 n}(s) \in R H_{\infty}$ and $C_{2 d}(s) \in R H_{\infty}$ be coprime factors of $C_{2}(s) C_{1 d}(s)$ satisfying

$$
C_{2}(s) C_{1 d}(s)=C_{2 n}(s) C_{2 d}^{-1}(s) .
$$

Combining (15) and (16), (11) can be obtained, where

$$
\begin{gathered}
Q_{0 n}(s)=\left\{Y(s) C_{1 n}(s)-X(s) C_{1 d}(s)\right\} C_{2 d}(s), \\
Q_{0 d}(s)=\left\{\widetilde{D}(s) C_{1 d}(s)+\widetilde{N}(s) C_{1 n}(s)\right\} C_{2 d}(s), \\
\widetilde{Q}(s)=C_{2 n}(s) .
\end{gathered}
$$

Note that $Q_{0 n}(s), Q_{0 d}(s)$ and $\widetilde{Q}(s)$, are $R H_{\infty}$ functions. As a consequence, the necessity can be proven if $Q(s)$ is a $H_{\infty}$ 
matrix function. According to (2), (9), and (13), $Q(s)$ can be rewritten as

$$
Q(s)=\{Y(s) C(s)-X(s)\}\{I+G(s) C(s)\}^{-1} \widetilde{D}^{-1}(s) .
$$

According to (12), the equation

$$
\widetilde{D}(s) \widetilde{Y}(s)+\widetilde{N}(s) \widetilde{X}(s)=I
$$

holds and $\widetilde{D}^{-1}(s)$ can be expressed as

$$
\widetilde{D}^{-1}(s)=\widetilde{Y}(s)+G(s) \widetilde{X}(s) .
$$

Subsequently,

$$
\begin{aligned}
Q(s)= & \{Y(s) C(s)-X(s)\}\{I+G(s) C(s)\}^{-1} \\
& \times\{\widetilde{Y}(s)+G(s) \widetilde{X}(s)\} \\
= & Y(s) C(s)\{I+G(s) C(s)\}^{-1} \widetilde{Y}(s) \\
& +Y(s) C(s)\{I+G(s) C(s)\}^{-1} G(s) \widetilde{X}(s) \\
& -X(s)\{I+G(s) C(s)\}^{-1} \widetilde{Y}(s) \\
& -X(s)\{I+G(s) C(s)\}^{-1} G(s) \widetilde{X}(s) .
\end{aligned}
$$

Because $C(s)$ stabilizes the closed system, $Q(s) \in H_{\infty}$ can be proven according to internal stability [15]. Therefore, the necessity is obtained.

Sufficiency. Suppose that there is $Q(s) \in H_{\infty}$ written by (11) such that (10b) stabilizes the closed-loop system in Figure 1. Substituting (10b) into (9) yields

$$
\begin{aligned}
C_{1}(s)= & \left\{\widetilde{X}(s) Q_{0 d}(s)+D(s) Q_{0 n}(s)\right\} \\
& \times\left\{\widetilde{Y}(s) Q_{0 d}(s)-N(s) Q_{0 n}(s)\right\}^{-1}, \\
C_{2}(s)= & \widetilde{Q}(s)\left\{\widetilde{Y}(s) Q_{0 d}(s)-N(s) Q_{0 n}(s)\right\}^{-1},
\end{aligned}
$$

respectively. Therefore, the sufficiency is obtained.

This completes the proof.

From Theorem 1, $\widetilde{Q}(s) \in R H_{\infty}, Q_{0 d}(s) \in R H_{\infty}$, and $Q_{0 n}(s) \in R H_{\infty}$ are the free parameters that need to be determined. In the following, the design methods for $\widetilde{Q}(s), Q_{0 d}(s)$, and $Q_{0 n}(s)$ are based on BMIs and the control characteristic.

3.2. Design of Free Parameters $Q_{0 d}(s)$ and $\widetilde{Q}(s)$. In $[12,13]$, the designs of the free parameters use the Nyquist stability criterion by manual examination, which is very difficult and inefficient. To resolve this problem, it was essential to establish an efficient and easy design method for the free parameters. In this subsection, an efficient design method for the free parameters using BMIs that can be solved with existing software is presented.

The first step is to design the free parameters $Q_{0 d}(s)$ and $\widetilde{Q}(s)$ such that $Q(s)$ belongs to $H_{\infty}$ on the basis of state-space description. According to (2) and (11), $Q(s)$ can be expressed as

$$
\begin{aligned}
& Q(s)=\left[Q_{0 d}(s)\right.Y(s) \widetilde{Q}(s)] Q_{f}(s), \\
& Q_{f}(s)=\left[\begin{array}{c}
I \\
C_{r}(s)
\end{array}\right]\left\{I+\left[\begin{array}{lll}
I & \widetilde{N}(s)
\end{array}\right]\right. \\
&\left.\times\left[\begin{array}{cc}
Q_{0 d}(s)-I & 0 \\
0 & \widetilde{Q}(s)
\end{array}\right]\left[\begin{array}{c}
I \\
C_{r}(s)
\end{array}\right]\right\}^{-1} .
\end{aligned}
$$

Because $Q_{0 n}(s) \in R H_{\infty}, Y(s) \in R H_{\infty}$, and $\widetilde{Q}(s) \in R H_{\infty}$, $Q(s) \in H_{\infty}$ means that the closed-loop system $Q_{f}(s)$ is stable. The problem of selecting the free parameters $Q_{0 d}(s) \in R H_{\infty}$ and $\widetilde{Q} \in R H_{\infty}$, while ensuring $Q(s) \in H_{\infty}$, is converted to design $Q_{0 d}(s) \in R H_{\infty}$ and $\widetilde{Q}(s) \in R H_{\infty}$, thus stabilizing the closed-loop system $Q_{f}(s)$. A general design method for $Q_{0 d}(s)$ and $\widetilde{Q}(s)$ was established, based on the state-space description and assuming a low-pass filter $q(s)$, as

$$
q(s)=\left[\begin{array}{c|c}
A_{l} & B_{l} \\
\hline C_{l} & 0
\end{array}\right] .
$$

Then, from Figure 1, the state-space description of $C_{r}(s)$ can be obtained as

$$
\begin{gathered}
\dot{x}_{r}(t)=A_{r_{0}} x_{r}(t)+A_{r_{1}} x_{r}(t-L)+B_{r} e(t), \\
z(t)=C_{r} x_{r}(t-L),
\end{gathered}
$$

where $x_{r}(t)$ is the state of $C_{r}(s)$,

$$
\begin{gathered}
A_{r_{0}}:=\left[\begin{array}{ccc}
A_{l} & & \\
& \ddots & \\
& & A_{l}
\end{array}\right], \\
A_{r_{1}}:=\left[\begin{array}{cccc}
B_{l} W_{1} C_{l} & \cdots & B_{l} W_{N-1} C_{l} & B_{l} W_{N} C_{l} \\
B_{l} C_{l} & & 0 \\
& \ddots & & \vdots \\
& & B_{l} C_{l} & 0
\end{array}\right], \\
B_{r}=\left[\begin{array}{llll}
B_{l}^{T} & 0 & \cdots & 0
\end{array}\right]^{T}, \quad C_{r}=\left[\begin{array}{llll}
W_{1} C_{l} & \cdots & W_{N} C_{l}
\end{array}\right] .
\end{gathered}
$$

Assume that the state-space descriptions of $Q_{0 d}(s), \widetilde{Q}(s)$, and $\widetilde{N}(s)$ are

$$
\begin{gathered}
Q_{0 d}(s)=\left[\begin{array}{c|c}
A_{0 d} & B_{0 d} \\
\hline C_{0 d} & D_{0 d}
\end{array}\right], \quad \widetilde{Q}(s)=\left[\begin{array}{c|c}
\widetilde{A}_{q} & \widetilde{B}_{q} \\
\hline \widetilde{C}_{q} & \widetilde{D}_{q}
\end{array}\right], \\
\widetilde{N}(s)=\left[\begin{array}{c|c}
\widetilde{A}_{n} & \widetilde{B}_{n} \\
\hline \widetilde{C}_{n} & \widetilde{D}_{n}
\end{array}\right],
\end{gathered}
$$

where $D_{0 d}$ has full column rank. Draw the configuration of $Q_{f}(s)$ in Figure 2 and the state-space description of the closed-loop system $Q_{f}(s)$ with $r(t) \equiv 0$ is given by

$$
\dot{x}(t)=A_{0} x(t)+A_{1} x(t-L),
$$




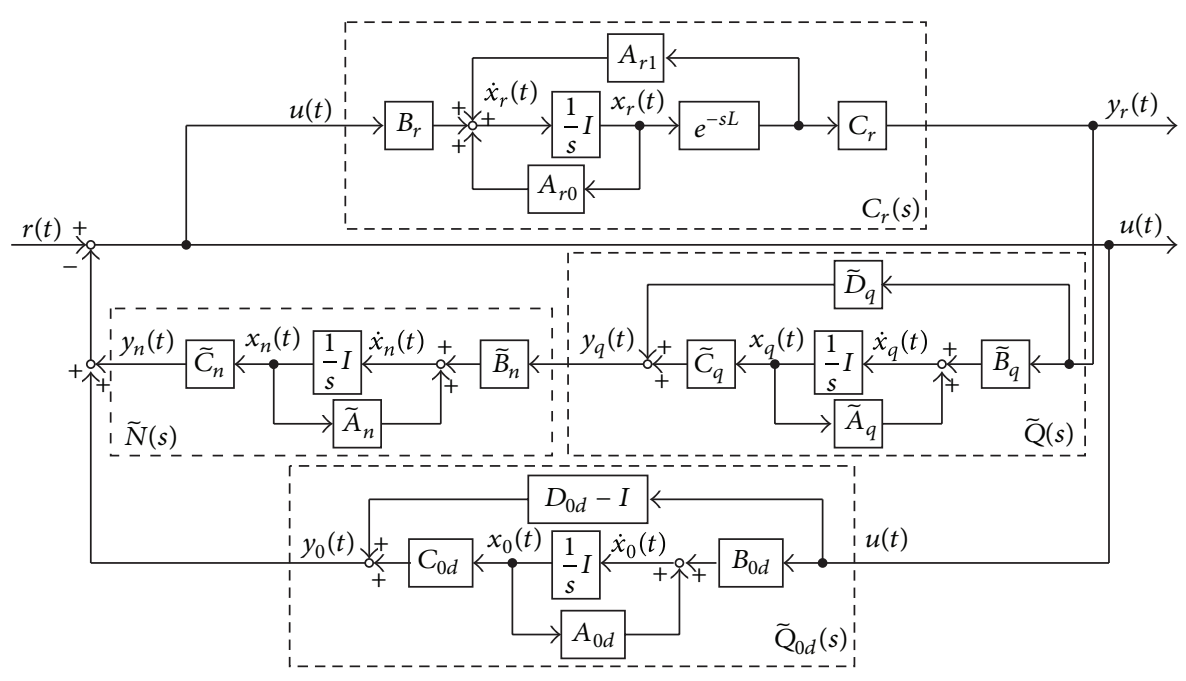

FIGURE 2: Configuration of $Q_{f}(s)$.

where

$$
\begin{gathered}
x(t):=\left[\begin{array}{llll}
x_{r}^{T}(t) & x_{n}^{T}(t) & x_{q}^{T}(t) & x_{0}^{T}(t)
\end{array}\right]^{T}, \\
A_{0}:=\left[\begin{array}{cccc}
A_{r 0} & -B_{r} D_{0 d}^{\dagger} \widetilde{C}_{n} & 0 & -B_{r} D_{0 d}^{\dagger} C_{0 d} \\
0 & \widetilde{A}_{n} & \widetilde{B}_{n} \widetilde{C}_{q} & 0 \\
0 & 0 & \widetilde{A}_{q} & 0 \\
0 & -B_{0 d} D_{0 d}^{\dagger} \widetilde{C}_{n} & 0 & A_{0 d}-B_{0 d} D_{0 d}^{\dagger} C_{0 d}
\end{array}\right], \\
A_{1}:=\left[\begin{array}{cccc}
A_{r 1} & 0 & 0 & 0 \\
\widetilde{B}_{n} \widetilde{D}_{q} C_{r 1} & 0 & 0 & 0 \\
\widetilde{B}_{q} C_{r 1} & 0 & 0 & 0 \\
0 & 0 & 0 & 0
\end{array}\right],
\end{gathered}
$$

$D_{0 d}^{\dagger}$ is the left pseudoinverse of $D_{0 d}$. The left pseudoinverse of $Q_{0 d}(s)$ is written as

$$
Q_{0 d}^{\dagger}(s)=\left[\begin{array}{c|c}
A_{0 d}-B_{0 d} D_{0 d}^{\dagger} C_{0 d} & -B_{0 d} D_{0 d}^{\dagger} \\
\hline D_{0 d}^{\dagger} C_{0 d} & D_{0 d}^{\dagger}
\end{array}\right]=\left[\begin{array}{c|c}
\bar{A}_{0 d} & \bar{B}_{0 d} \\
\hline \bar{C}_{0 d} & \bar{D}_{0 d}
\end{array}\right] .
$$

This means that the normal rank of $Q_{0 d}(s)$ is $p$ and $Q_{0 d}(s)$ could also be represented as

$$
Q_{0 d}(s)=\left[\begin{array}{c|c}
\bar{A}_{0 d}-\bar{B}_{0 d} D_{0 d} \bar{C}_{0 d} & -\bar{B}_{0 d} D_{0 d} \\
\hline D_{0 d} \bar{C}_{0 d} & D_{0 d}
\end{array}\right]=\left[\begin{array}{c|c}
A_{0 d} & B_{0 d} \\
\hline C_{0 d} & D_{0 d}
\end{array}\right] .
$$

Replacing $A_{0 d}-B_{0 d} D_{0 d}^{\dagger} C_{0 d},-B_{0 d} D_{0 d}^{\dagger}, D_{0 d}^{\dagger} C_{0 d}$, and $D_{0 d}^{\dagger}$, with $\bar{A}_{0 d}, \bar{B}_{0 d}, \bar{C}_{0 d}$, and $\bar{D}_{0 d}$ in (32), respectively, the system matrix $A_{0}$ is written as

$$
A_{0}=\left[\begin{array}{cccc}
A_{r 0} & -B_{r} \bar{D}_{0 d} \widetilde{C}_{n} & 0 & -B_{r} \bar{C}_{0 d} \\
0 & \widetilde{A}_{n} & \widetilde{B}_{n} \widetilde{C}_{q} & 0 \\
0 & 0 & \widetilde{A}_{q} & 0 \\
0 & \bar{B}_{0 d} \widetilde{C}_{n} & 0 & \bar{A}_{0 d}
\end{array}\right] .
$$

By employing the Lyapunov functional method to system (30), the following result can be derived.

Theorem 2. For system (30), if symmetric positive-definite matrices $P>0$ and $Q>0$ exist such that

$$
\Pi:=\left[\begin{array}{cc}
P A_{0}+A_{0}^{T} P+Q & P A_{1} \\
* & -Q
\end{array}\right]<0,
$$

then the time-delay system (30) is stable.

Proof. Choose the candidate Lyapunov functional to be

$$
V(x(t), t)=x^{T}(t) P x(t)+\int_{t-L}^{t} x^{T}(\theta) Q x(\theta) d \theta,
$$

where $P=P^{T}>0$ and $Q=Q^{T}>0$. Calculating the derivative of $V(t)$ for the time-delay system (30) yields

$$
\begin{aligned}
\dot{V}(x(t), t)= & 2 x^{T}(t) P \dot{x}(t)+x^{T}(t) Q x(t) \\
& -x^{T}(t-L) Q x(t-L) \\
= & 2 x^{T}(t) P\left(A_{0} x(t)+A_{1} x(t-L)\right) \\
:= & \xi^{T}(t) \Pi \xi(t),
\end{aligned}
$$

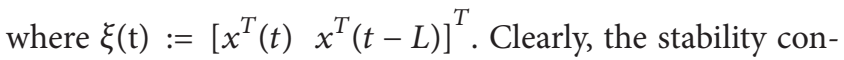
dition for the time-delay system (30) is

$$
\Pi<0 \text {. }
$$

This completes the proof.

Theorem 1 requires that $\widetilde{Q}(s)$ and $Q_{0 d}(s)$ are $R H_{\infty}$ matrix functions, which means that $\widetilde{Q}(s)$ and $Q_{0 d}(s)$ should be stable. According to Lyapunov functional theory, the BMI condition (35) contains the stability condition of $\widetilde{Q}(s)$. Hence, it is only 
necessary to provide the stability condition of $Q_{0 d}(s)$, and the following theorem will provide the stability condition in the form of BMIs for $Q_{0 d}(s)$.

Theorem 3. For system (30), if symmetric positive-definite matrices $P>0, Q>0$, and $X>0$ exist such that (35) and

$$
\Xi:=\left[\begin{array}{ccc}
X \bar{A}_{0 d}+\bar{A}_{0 d}^{T} X & X \bar{B}_{0 d} & \bar{C}_{0 d}^{T} \\
* & -I & 0 \\
* & * & -\bar{D}_{0 d} \bar{D}_{0 d}^{T}
\end{array}\right]<0
$$

hold, then the time-delay system (30) is stable and $Q_{0 d}(s) \in$ $R H_{\infty}$.

Proof. From Lyapunov functional theory, $Q_{0 d}(s)$ is a $R H_{\infty}$ matrix function if there exists a symmetric positive-definite matrix $X=X^{T}>0$ such that

$$
X A_{0 d}+A_{0 d}^{T} X<0
$$

which is equivalent to

$$
X \bar{A}_{0 d}-X \bar{B}_{0 d} D_{0 d} \bar{C}_{0 d}-\left(\bar{B}_{0 d} D_{0 d} \bar{C}_{0 d}\right)^{T} X+\bar{A}_{0 d}^{T} X<0 .
$$

According to the results in [16], a sufficient condition guaranteeing (41) is that there exists a positive number $\lambda>0$ such that

$$
\lambda X \bar{A}_{0 d}+\lambda A_{0 d}^{T} X+\lambda^{2} X \bar{B}_{0 d} \bar{B}_{0 d}^{T} X+\bar{C}_{0 d}^{T} D_{0 d}^{T} D_{0 d} \bar{C}_{0 d}<0 .
$$

Replacing $\lambda X$ and $D_{0 d}^{T} D_{0 d}$ with $X$ and $\left(\bar{D}_{0 d} \bar{D}_{0 d}^{T}\right)^{-1}$ and applying the Schur complement lemma show that (42) is equivalent to (39).

This completes the proof.

To avoid zero solutions, the initial transfer matrices for $\widetilde{Q}(s)$ and $Q_{0 d}^{\dagger}(s)$ are required. Without loss of generality, $\widetilde{Q}(s)$ and $Q_{0 d}^{\dagger}(s)$ should be represented as

$$
\begin{gathered}
\widetilde{Q}(s)=\left[\begin{array}{c|c}
\widetilde{A}_{q}+\Delta \widetilde{A}_{q} & \widetilde{B}_{q}+\Delta \widetilde{B}_{q} \\
\hline \widetilde{C}_{q}+\Delta \widetilde{C}_{q} & \widetilde{D}_{q}+\Delta \widetilde{D}_{q}
\end{array}\right], \\
Q_{0 d}^{\dagger}(s)=\left[\begin{array}{c|c}
\bar{A}_{0 d}+\Delta \bar{A}_{0 d} & \bar{B}_{0 d}+\Delta \bar{B}_{0 d} \\
\hline \bar{C}_{0 d}+\Delta \bar{C}_{0 d} & \bar{D}_{0 d}+\Delta \bar{D}_{0 d}
\end{array}\right],
\end{gathered}
$$

where $\widetilde{A}_{q}, \widetilde{B}_{q}, \widetilde{C}_{q}, \widetilde{D}_{q}, \bar{A}_{0 d}, \bar{B}_{0 d}, \bar{C}_{0 d}$, and $\bar{D}_{0 d}$ are the given initial matrices, and $\Delta \widetilde{A}_{q}, \Delta \widetilde{B}_{q}, \Delta \widetilde{C}_{q}, \Delta \widetilde{D}_{q}, \Delta \bar{A}_{0 d}, \Delta \bar{B}_{0 d}$, $\Delta \bar{C}_{0 d}$, and $\Delta \bar{D}_{0 d}$ are the unknown matrices that need to be determined. In the following theorem, modified stability conditions are proposed in the form of BMIs.

Theorem 4. For given initial matrix functions $\widetilde{Q}(s)$ and $Q_{0 d}^{\dagger}(s)$, if symmetric positive-definite matrices $P>0, Q>0$, and $X>0$ and appropriately dimensioned matrices $\Delta \widetilde{A}_{q}, \Delta \widetilde{B}_{q}$, $\Delta \widetilde{C}_{q}, \Delta \widetilde{D}_{q}, \Delta \bar{A}_{0 d}, \Delta \bar{B}_{0 d}, \Delta \bar{C}_{0 d}$, and $\Delta \bar{D}_{0 d}$ exist such that

$$
\begin{gathered}
{\left[\begin{array}{cc}
P\left(A_{0}+\Delta A_{0}\right)+\left(A_{0}^{T}+\Delta A_{0}^{T}\right) P+Q & P\left(\begin{array}{c}
\left.A_{1}+\Delta A_{1}\right) \\
*
\end{array}\right. \\
& -Q
\end{array}\right]<0,} \\
{\left[\begin{array}{ccc}
\Lambda & X\left(\bar{B}_{0 d}+\Delta \bar{B}_{0 d}\right) & \bar{C}_{0 d}^{T}+\Delta \bar{C}_{0 d}^{T} \\
* & -I & 0 \\
* & * & -\left(\bar{D}_{0 d}+\Delta \bar{D}_{0 d}\right)\left(\bar{D}_{0 d}+\Delta \bar{D}_{0 d}\right)^{T}
\end{array}\right]<0}
\end{gathered}
$$

hold, where $\Lambda, \Delta A_{0}$, and $A_{1}$ are defined as

$$
\begin{gathered}
\Lambda:=X\left(\bar{A}_{0 d}+\Delta \bar{A}_{0 d}\right)+\left(\bar{A}_{0 d}^{T}+\Delta \bar{A}_{0 d}^{T}\right) X, \\
\Delta A_{0}:=\left[\begin{array}{cccc}
0 & -B_{r} \Delta \bar{D}_{0 d} \widetilde{C}_{n} & 0 & -B_{r} \Delta \bar{C}_{0 d} \\
0 & 0 & \widetilde{B}_{n} \Delta \widetilde{C}_{q} & 0 \\
0 & 0 & \Delta \widetilde{A}_{q} & 0 \\
0 & \Delta \bar{B}_{0 d} \widetilde{C}_{n} & 0 & \Delta \bar{A}_{0 d}
\end{array}\right], \\
\Delta A_{1}:=\left[\begin{array}{ccccc}
0 & 0 & 0 & 0 \\
\widetilde{B}_{n} \Delta \widetilde{D}_{q} C_{r 1} & 0 & 0 & 0 \\
\Delta \widetilde{B}_{q} C_{r 1} & 0 & 0 & 0 \\
0 & 0 & 0 & 0
\end{array}\right],
\end{gathered}
$$

then the time-delay system (30) is stable and $\mathrm{Q}_{0 d}(s)$ is $R H_{\infty}$.

Proof. Replacing matrices $\widetilde{A}_{q}, \widetilde{B}_{q}, \widetilde{C}_{q}, \widetilde{D}_{q}, \bar{A}_{0 d}, \bar{B}_{0 d}, \bar{C}_{0 d}$, and $\bar{D}_{0 d}$ with matrices $\widetilde{A}_{q}+\Delta \widetilde{A}_{q}, \widetilde{B}_{q}+\Delta \widetilde{B}_{q}, \widetilde{C}_{q}+\Delta \widetilde{C}_{q}, \widetilde{D}_{q}+\Delta \widetilde{D}_{q}$, $\bar{A}_{0 d}+\Delta \bar{A}_{0 d}, \bar{B}_{0 d}+\Delta \bar{B}_{0 d}, \bar{C}_{0 d}+\Delta \bar{C}_{0 d}$, and $\bar{D}_{0 d}+\Delta \bar{D}_{0 d}$, respectively, in (35) and (39), the constraints (44) can be obtained.

This completes this proof.

Constraints (44) are the BMIs that can be solved by BMI solvers, such as PENBMI [17] and bmibnb in YALMIP [18].

3.3. Design of Free Parameter $Q_{0 n}(s)$. As mentioned, the objective of this paper is to develop a new design method that results in a closed-loop system in Figure 1 that is robustly stable and has high control precision for reference inputs with uncertain period-time. Hence, the free parameter $Q_{0 n}(s)$ should be designed after the control characteristic. The output sensitivity matrix function is defined as the transfer matrix function from the reference input $r(t)$ to the tracking error $e(t)$ :

$$
S(s):=(I+G(s) C(s))^{-1} .
$$

Having good tracking performance of the closed-loop system requires that

$$
\bar{\sigma}\{S(s)\}=\bar{\sigma}\left\{(I+G(s) C(s))^{-1}\right\}
$$


be as small as possible [15]. From (12), (10b), and (11), the sensitivity matrix function is written as

$$
\begin{aligned}
S(s)= & \left\{\widetilde{Y}(s) Q_{0 d}(s)-N(s) Q_{0 n}(s)\right\} \\
& \times\left\{Q_{0 d}(s)+\left(\widetilde{N}(s) \widetilde{Q}(s)-Q_{0 d}(s)\right) H(s)\right\}^{-1} \\
& \times(I-H(s)) \widetilde{D}(s),
\end{aligned}
$$

and the largest singular value of the sensitivity matrix function is

$$
\begin{aligned}
\bar{\sigma}\{S(s)\} \leq & \bar{\sigma}\left\{\widetilde{Y}(s) Q_{0 d}(s)-N(s) Q_{0 n}(s)\right\} \\
& \times \bar{\sigma}\left\{\left(Q_{0 d}(s)+\left(\widetilde{N}(s) \widetilde{Q}(s)-Q_{0 d}(s)\right) H(s)\right)^{-1}\right\} \\
& \times \bar{\sigma}\{(I-H(s))\} \bar{\sigma}\{\widetilde{D}(s)\} .
\end{aligned}
$$

Because of $Q(s) \in H_{\infty}, \widetilde{D}(s) \in R H_{\infty}$, and $q(s)$ satisfying Assumptions 1 and 2, when the reference signal $r(t)$ is assumed to be a periodic signal with a known and fixed period-time $L$, the output $y(t)$ follows the reference input $r(t)$ with a small steady-state error because of

$$
\bar{\sigma}\left\{I-H\left(j w_{l}\right)\right\} \simeq 0, \quad(l=0, \ldots, \hbar) .
$$

However, for the reference input with unknown period-time, the frequency components $\bar{\omega}_{k}(k=0, \ldots, h)$ of the reference input are not equal to $\omega_{l}(l=1, \ldots, \hbar)$, which yields

$$
\bar{\sigma}\left\{S\left(j \bar{\omega}_{k}\right)\right\} \neq 0, \quad(k=0, \ldots, h),
$$

where $\bar{\omega}_{k}(k=0, \ldots, h)$ are defined as

$$
\bar{\omega}_{k}:=\frac{2 k \pi}{L(1+\delta)}, \quad|\delta| \leq \Delta<\infty
$$

for $k=0, \ldots, h$. To obtain a small steady-state error, $Q_{0 n}(s) \in$ $R H_{\infty}$ will be selected such that

$$
\begin{array}{r}
\bar{\sigma}\left\{\tilde{Y}\left(j \bar{\omega}_{k}\right) Q_{0 d}\left(j \bar{\omega}_{k}\right)-N\left(j \bar{\omega}_{k}\right) Q_{0 n}\left(j \bar{\omega}_{k}\right)\right\} \simeq 0 \\
(i=1, \ldots, h) .
\end{array}
$$

Hereby, the design method for $Q_{0 n}(s)$ is detailed depending upon the sensitivity function. According to [19], $N(s)$ is strictly proper when $G(s)$ is a strictly proper system. $N(s)$ is factorized as

$$
N(s)=N_{i}(s) N_{o}(s)
$$

where $N_{i}(s)$ is a square inner matrix function with $N_{i}(0)=I$ and $N_{o}(s)$ is an outer matrix function $[15,20]$. Because $N_{o}(s)$ is an outer matrix, there exists a stable $m \times p$ matrix $N_{o}^{+}(s)$ such that

$$
N_{o}(s) N_{o}^{+}(s)=I \text {. }
$$

For this reason, $Q_{0 n}(s)$ and $\bar{q}(s)$ are chosen by

$$
\begin{gathered}
Q_{0 n}(s)=N_{o}^{+}(s) \bar{q}(s) \tilde{Y}(s) Q_{0 d}(s), \\
\bar{q}(s)=\operatorname{diag}\left\{\frac{1}{\left(1+\tau_{1} s\right)^{\alpha_{1}}}, \ldots, \frac{1}{\left(1+\tau_{p} s\right)^{\alpha_{p}}}\right\} \in R H_{\infty},
\end{gathered}
$$

where $\alpha_{i}(i=1, \ldots, p)$ is a positive integer to make $Q_{0 n}(s)$ proper; then,

$$
\tilde{Y}(s) Q_{0 d}(s)-N(s) Q_{0 n}(s)=\left(I-N_{i}(s) \bar{q}(s)\right) \tilde{Y}(s) Q_{0 d}(s) .
$$

Therefore, when $\bar{q}(s)$ is chosen to satisfy

$$
\bar{\sigma}\left\{I-N_{i}\left(j \bar{\omega}_{k}\right) \bar{q}\left(j \bar{\omega}_{k}\right)\right\} \simeq 0, \quad(k=0, \ldots, h),
$$

the output $y(t)$ follows the reference input $r(t)$ with a small steady-state error that is caused by

$$
\bar{\sigma}\left\{S\left(j \bar{\omega}_{k}\right)\right\} \simeq 0
$$

for $k=0, \ldots, h$. The designs for $N_{i}(s), N_{o}(s)$, and $\bar{q}(s)$ are described in detail in [21-24].

Hence, by applying this design method to the free parameters, both stability and good control performance can be guaranteed for the high-order modified repetitive control system. Consequently, the following procedures to design the stabilizing high-order modified repetitive control system are provided.

\subsubsection{Procedure}

Step 1. Select the constant $N$, with low-pass filter $q(s)$, and calculate the $N$ weighting factors $W_{i}(i=1, \ldots, N)$ according to $[5,7,10]$.

Step 2. According to [19], obtain the coprime factors $N(s)$, $D(s), \widetilde{N}(s)$, and $\widetilde{D}(s)$ and parameters $X(s), Y(s), \widetilde{X}(s)$, and $\widetilde{Y}(s)$.

Step 3. Settle the initial matrix functions $\widetilde{Q}(s) \in R H_{\infty}$ and $\mathrm{Q}_{0 d}^{\dagger}(s) \in R H_{\infty}$, and obtain the state-space description of the closed-loop system (30).

Step 4. Solve the feasible problems (44) to obtain $\Delta \widetilde{A}_{q}, \Delta \widetilde{B}_{q}$, $\Delta \widetilde{C}_{q}, \Delta \widetilde{D}_{q}, \Delta \bar{A}_{0 d}, \Delta \bar{B}_{0 d}, \Delta \bar{C}_{0 d}$, and $\Delta \bar{D}_{0 d}$.

Step 5. Replace matrices $\widetilde{A}_{q}, \widetilde{B}_{q}, \widetilde{C}_{q}, \widetilde{D}_{q}, \bar{A}_{0 d}, \bar{B}_{0 d}, \bar{C}_{0 d}$, and $\bar{D}_{0 d}$ with matrices $\widetilde{A}_{q}+\Delta \widetilde{A}_{q}, \widetilde{B}_{q}+\Delta \widetilde{B}_{q}, \widetilde{C}_{q}+\Delta \widetilde{C}_{q}, \widetilde{D}_{q}+\Delta \widetilde{D}_{q}$, $\bar{A}_{0 d}+\Delta \bar{A}_{0 d}, \bar{B}_{0 d}+\Delta \bar{B}_{0 d}, \bar{C}_{0 d}+\Delta \bar{C}_{0 d}$, and $\bar{D}_{0 d}+\Delta \bar{D}_{0 d}$, respectively. In addition, calculate the matrices $A_{0 d}, B_{0 d}, C_{0 d}$, and $D_{0 d}$ using (33).

Step 6. To obtain good tracking performance, the low-pass filter $\bar{q}(s)$ is settled to be the form of (59) with $\bar{q}(0)=I$ and to satisfy (61). 
Step 7. Using the above parameters, the high-order modified repetitive controller $C(s)$ in (9) can be obtained for system (8), where $C_{1}(s)$ and $C_{2}(s)$ are written as $(22)$ and (23), respectively.

The design procedure proposed in this section is applicable for both SISO linear systems and multiple-input/multipleoutput (MIMO) linear systems by simply modifying the dimensions of some matrices.

\section{Numerical Examples}

In this section, numerical examples are generated to illustrate the validity and effectiveness of the proposed approach. Consider a SISO strictly proper plant $G(s)$ with the following parameters:

$$
A_{p}=\left[\begin{array}{cc}
1 & 3 \\
2 & -5
\end{array}\right], \quad B_{p}=\left[\begin{array}{l}
1 \\
0
\end{array}\right], \quad C_{p}=\left[\begin{array}{ll}
1 & 0
\end{array}\right] .
$$

For this unstable control plant, it is easy to verify that the pairs $\left(A_{p}, B_{p}\right)$ and $\left(A_{p}, C_{p}\right)$ are controllable and observable, respectively. According to [19], choose the $F$ and $L$ as follows:

$$
F=\left[\begin{array}{ll}
-3 & -4
\end{array}\right], \quad L=\left[\begin{array}{ll}
-1 & -4
\end{array}\right]^{T} \text {. }
$$

Then, the parameters $N(s), D(s), \widetilde{N}(s), \widetilde{D}(s), X(s), Y(s), \widetilde{X}(s)$, and $\widetilde{Y}(s)$ are given by

$$
\begin{aligned}
& {\left[\begin{array}{cc}
-D(s) & \widetilde{X}(s) \\
N(s) & \widetilde{Y}(s)
\end{array}\right]=\left[\begin{array}{cc}
-\frac{s^{2}+4 s-11}{s^{2}+7 s+12} & \frac{19 s+43}{s^{2}+7 s+12} \\
\frac{s+5}{s^{2}+7 s+12} & \frac{s^{2}+8 s+13}{s^{2}+7 s+12}
\end{array}\right],} \\
& {\left[\begin{array}{cc}
Y(s) & X(s) \\
\widetilde{N}(s) & -\widetilde{D}(s)
\end{array}\right]=\left[\begin{array}{cc}
\frac{s^{2}+8 s+13}{s^{2}+5 s+6} & \frac{19 s+43}{s^{2}+5 s+6} \\
\frac{s+5}{s^{2}+5 s+6} & -\frac{s^{2}+4 s-11}{s^{2}+5 s+6}
\end{array}\right] .}
\end{aligned}
$$

In this paper, to show the generality of this design method and obtain the weighting factors conveniently, $N=5$ was chosen. As is well known, the low-pass filter $q(s)$ has a bandwidth restriction for a nonminimum phase plant. Here, we chose the robust low-pass filter $q(s)$ to be

$$
q(s)=\frac{1}{0.01 s+1} .
$$

Considering improvements to the control precision for intermediate frequencies and that the low-pass filter $\bar{q}(s)$ has no restriction on bandwidth, set $\bar{q}(s)$ as

$$
\bar{q}(s)=\frac{1}{0.001 s+1} .
$$

To improve the system sensitivity at intermediate frequencies, we chose the high-order repetitive controller as noise robust [10]. The corresponding weighting factors $W_{i}(i=1, \ldots, 5)$ could then be obtained directly from $[10]$ :

$$
W=\left[\begin{array}{lllll}
\frac{5}{15} & \frac{4}{15} & \frac{3}{15} & \frac{2}{15} & \frac{1}{15}
\end{array}\right]
$$

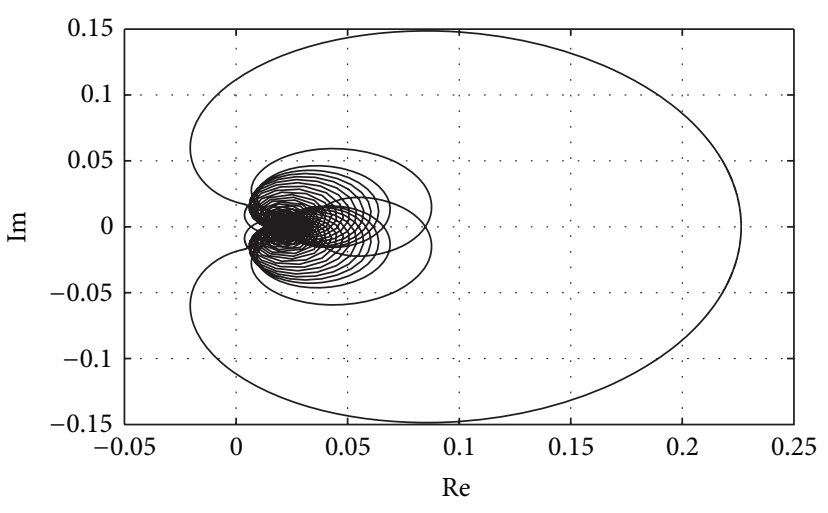

Figure 3: Nyquist plot of $\operatorname{det}\left\{Q_{0 d}(s)+\left(\widetilde{N}(s) \widetilde{Q}(s)-Q_{0 d}(s)\right) H(s)\right\}$.

The initial matrix functions $\widetilde{Q}(s)$ and $Q_{0 d}^{-1}(s)$ were set as

$$
\widetilde{Q}(s)=\frac{s^{2}+5 s+6}{s^{2}+15 s+50}, \quad Q_{0 d}^{-1}(s)=\frac{s^{2}+5 s+16}{s^{2}+5 s+6} .
$$

Then, the feasible problem is solved using the PENBMI solver to obtain $\widetilde{Q}(s)$ and $Q_{0 d}(s)$ as

$$
\begin{gathered}
\widetilde{Q}(s)=\frac{4.5331 s^{2}+122.5857 s+424.0851}{s^{2}+90.9312 s+1561.1741}, \\
Q_{0 d}(s)=\frac{0.1656 s^{2}+6.4867 s+63.0101}{s^{2}+32.3705 s+219.2992} .
\end{gathered}
$$

Because $N(s)$ is the minimum phase, the inner-outer factorization of $N(s)$ can be set as

$$
N(s)=N_{i}(s) N_{o}(s),
$$

where $N_{i}(s)=I$ and $N_{o}(s)=N(s)$. Then, $Q_{0 n}(s)$ is

$$
Q_{0 n}(s)=N^{-1}(s) \bar{q}(s) \tilde{Y}(s) Q_{0 d}(s) .
$$

According to the Nyquist stability criterion, $Q_{0 d}(s) \in$ $R H_{\infty}, \widetilde{Q}(s) \in R H_{\infty}$, and $\widetilde{N}(s) \in R H_{\infty}$; then, $Q(s) \in H_{\infty}$ means that the Nyquist plot of $\operatorname{det}\left\{Q_{0 d}(s)+(\widetilde{N}(s) \widetilde{Q}(s)-\right.$ $\left.\left.Q_{0 d}(s)\right) H(s)\right\}$ does not encircle the origin $(0,0)$. Obviously, Figure 3 illustrates that $Q(s) \in H_{\infty}$. This demonstrates that the design method for the free parameters $\widetilde{Q}(s)$ and $Q_{0 d}(s)$ is much more effective than that in $[12,13]$.

Using the above-mentioned parameters, the stabilizing controller $C(s)$, which contains a high-order modified repetitive control structure in (9), was obtained. To demonstrate its effectiveness, sensitivity function plots were made and simulations were conducted for the reference inputs:

$$
r(t)=2 \sin \left(\frac{2 \pi}{L+\Delta L} t\right), \quad L=1[\mathrm{sec}] .
$$

The magnitude of the resulting sensitivity function is plotted in Figure 4, for a system with and without repetitive control. From this figure, it can be concluded that the high-order controller provided a better control performance when the 


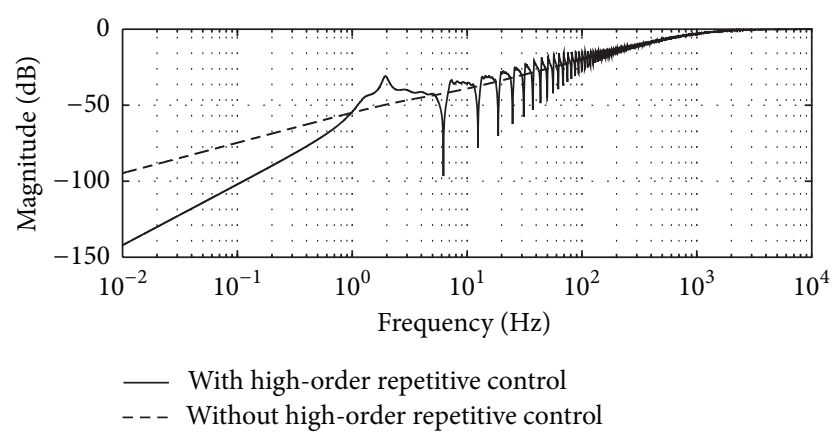

FIGURE 4: Sensitivity function of the high-order repetitive control system.

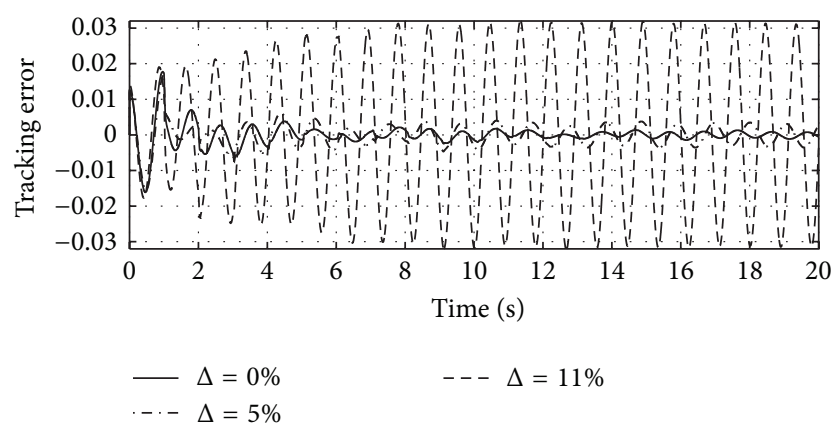

FIGURE 5: Tracking errors for the references with uncertain periodtime.

period-time had a limited uncertainty. When the reference had intermediate frequency components, the control performance became worse than that without the repetitive controller. However, the control performance at the intermediate frequencies was improved by modifying the cutoff frequency of the low-pass filter $\bar{q}(s)$.

The simulation results using our method are shown in Figure 5. Clearly, the high-order modified repetitive control system was stable. For the period-time without variation, the largest steady-state peak-to-peak relative error was as small as $0.05 \%$, and this system entered the steady state in the 11th period. When the period-time had an uncertainty level $\Delta$ of $5 \%$, the largest steady-state peak-to-peak relative error was $0.15 \%$. This result shows that for a certain vibration in period-time, the high-order modified repetitive controller continued to provide high control precision. As seen in Figure 4 , when $\Delta=11 \%$, the largest steady-state peak-topeak relative error of this control system increases to $1.6 \%$. This tracking error is small and can be further reduced by modifying the cutoff frequency of the low-pass filter $\bar{q}(s)$ because it has no bandwidth restriction. The results shown in Figure 5 demonstrate that the design method presented here provided not only good stability, but also satisfactory tracking performance for the reference inputs with uncertain period-time. Compared with conventional design methods, the design method proposed in this paper widens the range of the uncertainty in period-time and has a wider range of practical applications, which makes it very practical.

\section{Conclusion}

This paper presents a new design method for high-order modified repetitive control systems for periodic reference inputs with uncertain period-time. A high-order modified repetitive control system was established and the parametrization of all stabilizing controllers was derived based on the YKP. Moreover, the constraint conditions of free parameters were converted into stability conditions in the form of BMIs, which can be solved using existing software. In addition, the control precision of this closed-loop control system was guaranteed by analyzing its control characteristic. From the simulation results, we found that the high-order repetitive controller provided high control precision with a certain vibration in period-time and that the control precision at intermediate frequencies could be satisfied by modifying the cutoff frequency of low-pass filters in compensators.

However, the design of this high-order modified repetitive control system was subject to a control plant without uncertainty and disturbance, because the low-pass filter that was used to specify the intermediate frequencies had bandwidth restrictions to address the problem of robust stabilization. For this reason, the control precision will be degraded and the uncertain range in period-time will be narrowed. This issue requires further attention, particularly in cases where there exist uncertainties in the plant. In such cases, the optimal performance needs to be considered, as do the largest cutoff frequencies and widening of the uncertain range in the period-time.

\section{Acknowledgments}

The first author would like to express his gratitude to the China Scholarship Council (CSC) for its support and scholarship. The authors gratefully acknowledge the reviewers for their helpful comments and suggestions, which have improved the presentation.

\section{References}

[1] S. Hara, Y. Yamamoto, T. Omata, and M. Nakano, "Repetitive control system: a new type servo system for periodic exogenous signals," IEEE Transactions on Automatic Control, vol. 33, no. 7, pp. 659-668, 1988.

[2] B. A. Francis and W. M. Wonham, "The internal model principle for linear multivariable regulators," Applied Mathematics of Optimization, vol. 2, no. 2, pp. 170-194, 1975.

[3] B. Mahawan and Z. H. Luo, "Repetitive control of tracking systems with time-varying periodic references," International Journal of Control, vol. 73, no. 1, pp. 1-10, 2000.

[4] J. S. Freudenberg and D. P. Looze, "Right half plane poles and zeros and design tradeoffs in feedback systems," IEEE Transactions on Automatic Control, vol. 30, no. 6, pp. 555-565, 1985.

[5] G. Pipeleers, B. Demeulenaere, J. De Schutter, and J. Swevers, "Robust high-order repetitive control: optimal performance trade-offs," Automatica, vol. 44, no. 10, pp. 2628-2634, 2008.

[6] T. Inoue, "Practical repetitive control system design," in Proceedings of the 29th IEEE Conference on Decision and Control, pp. 1673-1678, Honolulu, Hawaii, USA, December 1990. 
[7] W. S. Chang, I. H. Suh, and T. W. Kim, "Analysis and design of two types of digital repetitive control systems," Automatica, vol. 31, no. 5, pp. 741-746, 1995.

[8] M. Steinbuch, "Repetitive control for systems with uncertain period-time," Automatica, vol. 38, no. 12, pp. 2103-2109, 2002.

[9] M. Steinbuch, S. Weiland, J. D. Van Eerenbeemt, and T. Singh, "On noise- and period-time sensitivity in high order repetitive control," in Proceedings of the 43rd IEEE Conference on Decision and Control (CDC '04), pp. 1295-1300, Atlantis, Paradise Islands, Bahamas, December 2004.

[10] M. Steinbuch, S. Weiland, and T. Singh, "Design of noise and period-time robust high-order repetitive control, with application to optical storage," Automatica, vol. 43, no. 12, pp. 20862095, 2007.

[11] G. Pipeleers, B. Demeulenaere, J. De Schutter, and J. Swevers, "Generalised repetitive control: relaxing the period-delay-based structure," IET Control Theory \& Applications, vol. 3, no. 11, pp. 1528-1536, 2009.

[12] K. Yamada, N. Li, M. Kobayashi, and H. Takenaga, "A design method for simple multi-period repetitive controllers for timedelay plants," International Journal of Innovative Computing, Information and Control, vol. 5, no. 10, pp. 3313-3328, 2009.

[13] K. Yamada, N. Nakazawa, I. Murakami et al., "A design method for two-degree-of-freedom multi-period repetitive control systems," Key Engineering Materials, vol. 459, pp. 194-210, 2011.

[14] M. Vidyasagar, "Control system synthesis: a factorization approach, part II," Synthesis Lectures on Control and Mechatronics, vol. 2, no. 1, pp. 1-227, 2011.

[15] K. Zhou, J. C. Doyle, and K. Glover, Robust and Optimal Control, Prentice-Hall, Upper Saddle River, NJ, USA, 1996.

[16] L. Xie, "Output feedback $H_{\infty}$ control of systems with parameter uncertainty," International Journal of Control, vol. 63, no. 4, pp. 741-750, 1996.

[17] M. Kocvara and M. Stingl, "PENBMI user's guide (Version 2.1)," 2005.

[18] J. Löfberg, "YALMIP: A toolbox for modeling and optimization in MATLAB," in Proceedings of IEEE International Symposium on Computer Aided Control System Design, pp. 284-289, Taipei, Taiwan, September 2004.

[19] C. N. Nett, C. A. Jacobson, and M. J. Balas, "A connection between state-space and doubly coprime fractional representations," IEEE Transactions on Automatic Control, vol. 29, no. 9, pp. 831-834, 1984.

[20] G. Gu, "Inner-outer factorization for strictly proper transfer matrices," IEEE Transactions on Automatic Control, vol. 47, no. 11, pp. 1915-1919, 2002.

[21] K. Yamada and K. Watanabe, "A state space design method of stable filtered inverse system," Transactions of the Society of Instrument and Control Engineers, vol. 32, no. 6, pp. 862-870, 1996.

[22] K. Yamada, K. Watanabe, and Z. B. Shu, "A state space design method of stable filtered inverse systems and its application to $\mathrm{H}_{2}$ suboptimal internal model control," in Proceedings of International Federation of Automatic Control World Congress, pp. 379-382, San Francisco, Calif, USA, 1996.

[23] K. Yamada and W. Kinoshita, "New design method for stable filtered inverse systems," in Proceedings of the American Control Conference, pp. 4738-4743, Anchorage, Alaska, USA, May 2002.

[24] K. Yamada and W. Kinoshita, "New state space design method of stable filtered inverse systems and their application," Transactions of the Institute of System, Control and Information Engineers, vol. 16, no. 2, pp. 85-93, 2003. 


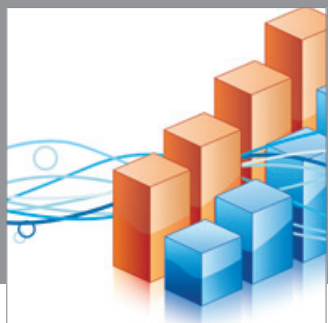

Advances in

Operations Research

mansans

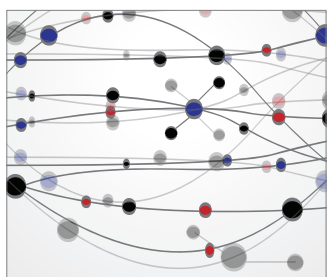

The Scientific World Journal
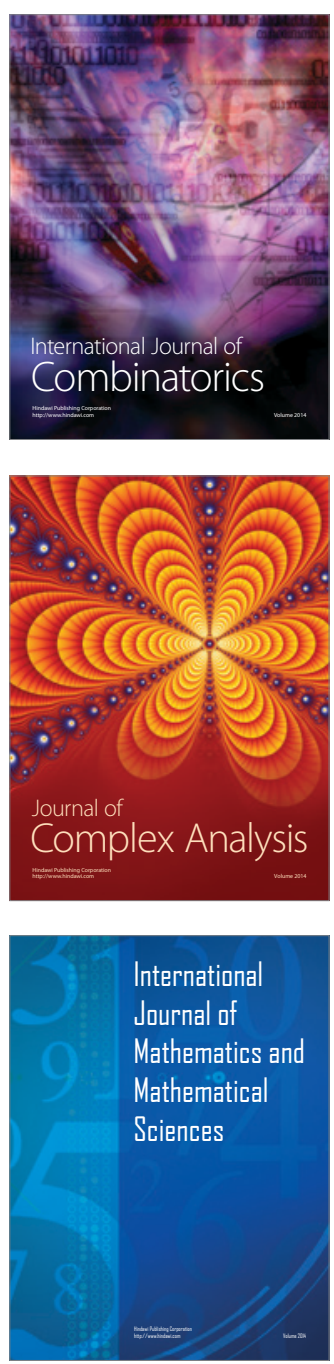
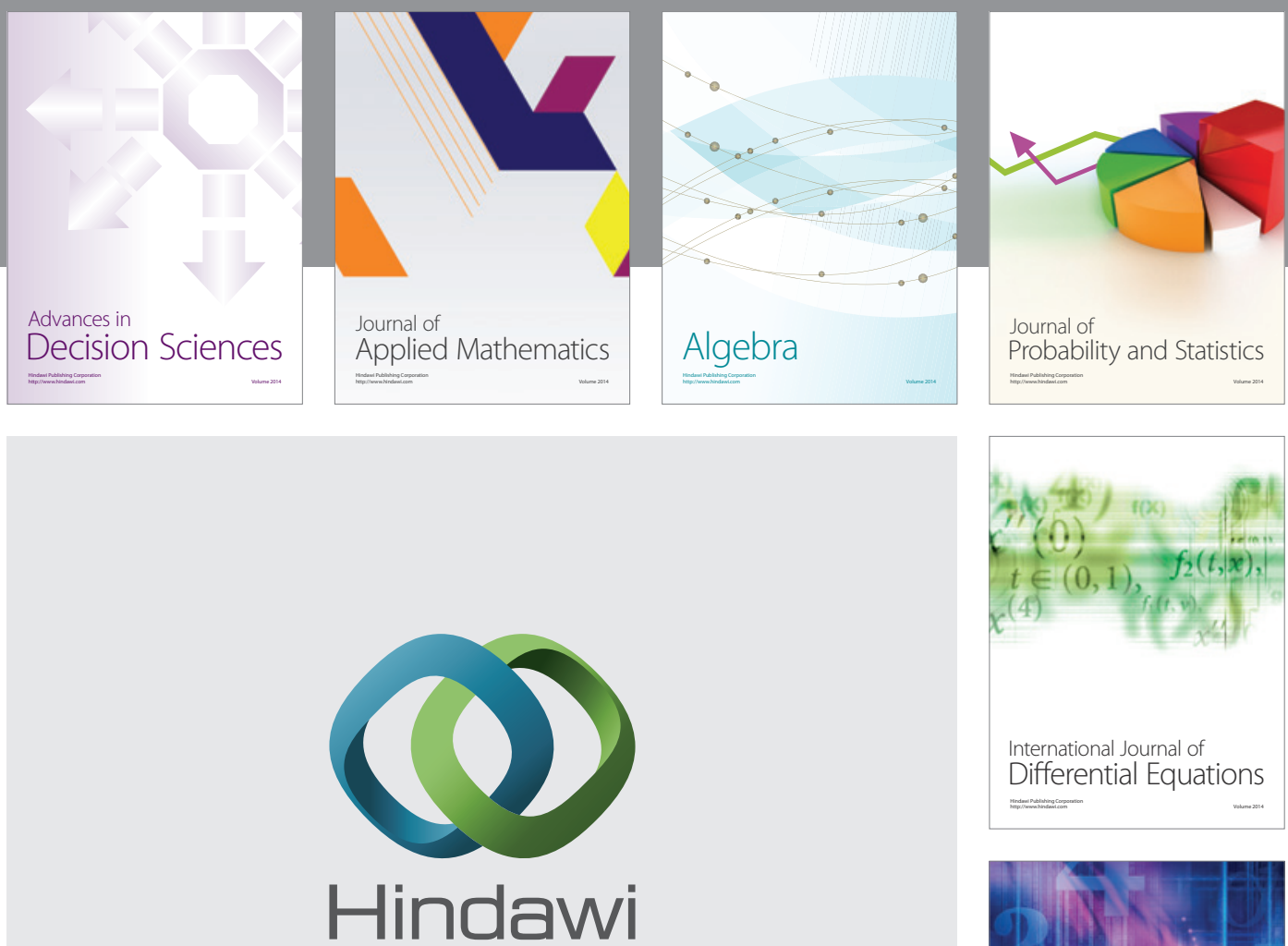

Submit your manuscripts at http://www.hindawi.com
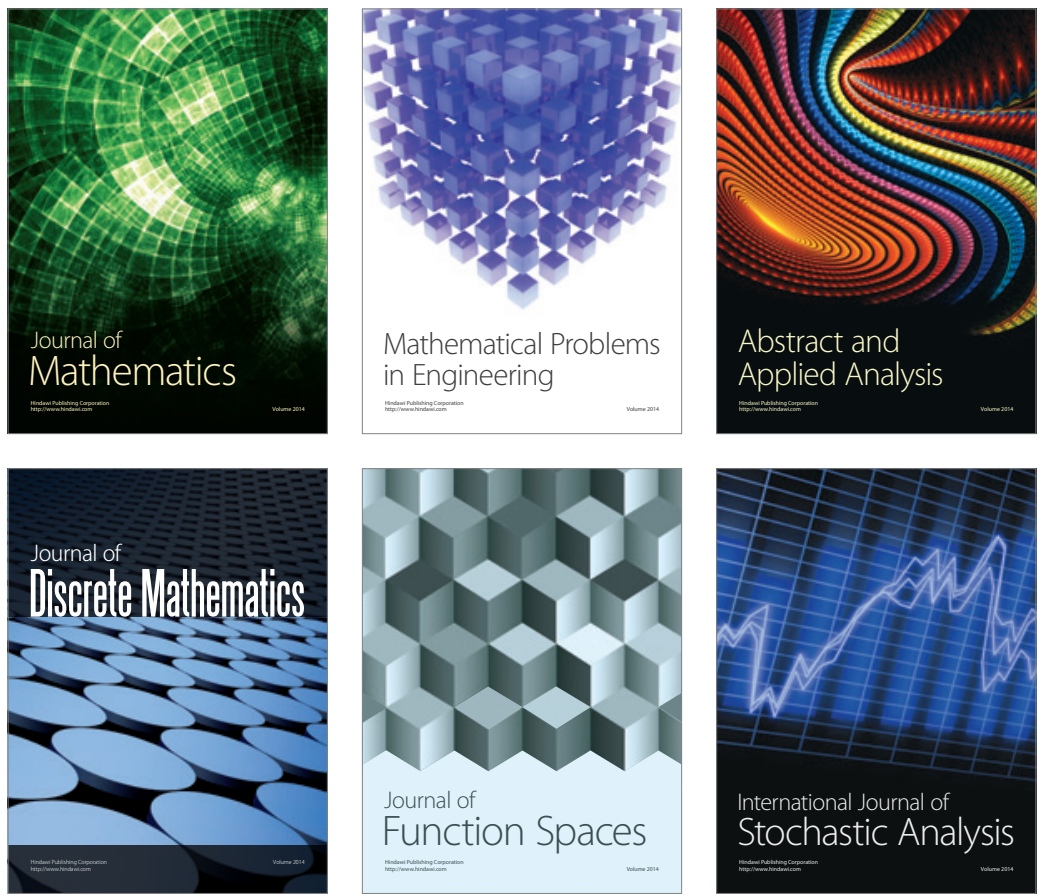

Journal of

Function Spaces

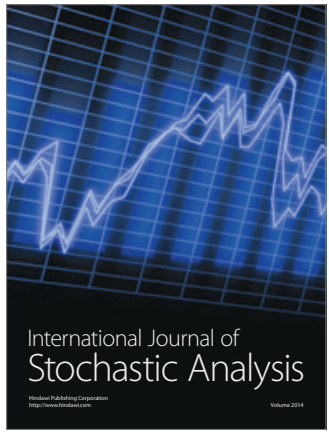

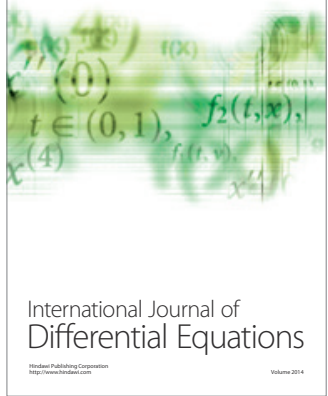
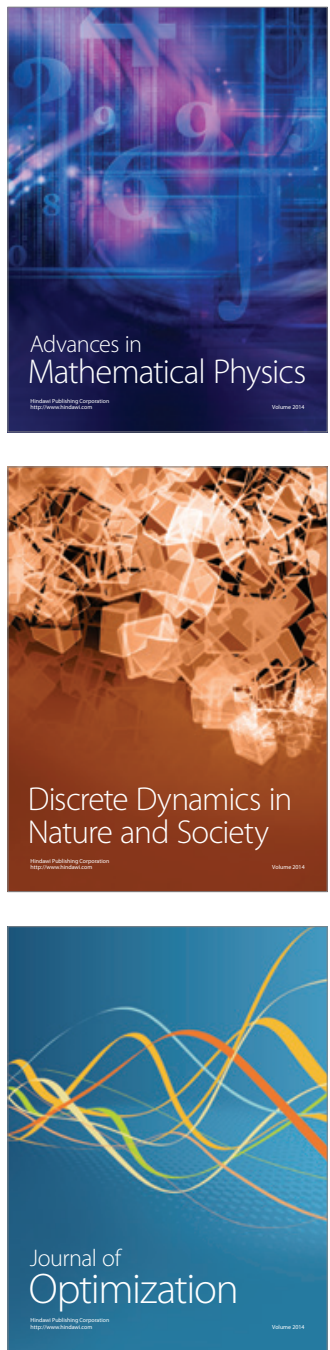